\title{
Elevation Spatial Variation Error Compensation in Complex Scene and Elevation Inversion by Autofocus Method in GEO SAR
}

\author{
Faguang Chang $\mathbb{D}^{D}$, Dexin Li *, Zhen Dong, Yang Huang and Zhihua He
}

check for updates

Citation: Chang, F.; Li, D.; Dong, Z.; Huang, Y.; He, Z. Elevation Spatial Variation Error Compensation in Complex Scene and Elevation Inversion by Autofocus Method in GEO SAR. Remote Sens. 2021, 13, 2916. https://doi.org/10.3390/rs13152916

Academic Editors: Andrzej Stateczny, Witold Kazimierski and

Krzysztof Kulpa

Received: 15 June 2021

Accepted: 19 July 2021

Published: 24 July 2021

Publisher's Note: MDPI stays neutra with regard to jurisdictional claims in published maps and institutional affiliations.

Copyright: (c) 2021 by the authors. Licensee MDPI, Basel, Switzerland. This article is an open access article distributed under the terms and conditions of the Creative Commons Attribution (CC BY) license (https:/ / creativecommons.org/licenses/by/ $4.0 /)$.
College of Electronic Science and Technology, National University of Defense Technology, No. 109 Deya Road, Changsha 410073, China; cfg@nudt.edu.cn (F.C.); dongzhen@nudt.edu.cn (Z.D.); huangyang18@nudt.edu.cn (Y.H.); zhihuahe@nudt.edu.cn (Z.H.)

* Correspondence: lidexi@nudt.edu.cn

\begin{abstract}
Due to the high altitude of geosynchronous synthetic aperture radar (GEO SAR), its synthetic aperture time can reach up to several hundred seconds, and its revisit cycle is very short, which makes it of great application worth in the remote sensing field, such as in disaster monitoring and vegetation measurements. However, because of the elevation of the target, elevation spatial variation error is caused in the GEO SAR imaging. In this paper, we focus on the compensation of the elevation space-variant error in the fast variant part with the autofocus method and utilize the error to carry out elevation inversing in complex scenes. For a complex scene, it can be broken down into a slow variant slope and the remaining fast variant part. First, the phase error caused by the elevation spatial variation is analyzed. Second, the spatial variant error caused by the slowly variant slope is compensated with the improved imaging algorithm. The error caused by the remaining fast variable part is the focus of this paper. We propose a block map-drift phase gradient autofocus (block-MD-PGA) algorithm to compensate for the random phase error part. By dividing sub-blocks reasonably, the elevation spatial variant error is compensated for by an autofocus algorithm in each sub-block. Because the errors of different elevations are diverse, the proposed algorithm is suitable for the scene where the target elevations are almost the same after the sub-blocks are divided. Third, the phase error obtained by the autofocus method is used to inverse the target elevation. Finally, simulations with dot-matrix targets and targets based on the high-resolution TerraSAR-X image verify the excellent effect of the proposed method and the accuracy of the elevation inversion.
\end{abstract}

Keywords: geosynchronous synthetic aperture radar (GEO SAR); fast variant part elevation spatial variant error; autofocus method; elevation inversion

\section{Introduction}

Since K. Tomiyasu first proposed the concept of Geosynchronous synthetic aperture radar (GEO SAR) and performed a preliminary and approximate analysis assuming a particular orbit in 1978, it has quickly attracted the attention of many researchers [1]. GEO SAR has many unique advantages, such as high resolution and being unaffected by national boundaries and meteorological conditions. GEO SAR has bright application prospects in many significant research fields due to its ultra-long synthetic aperture time, wide swath, long dwell time and short revisit time, such as disaster monitoring and vegetation measurements [2,3]. By setting different orbit parameters (eccentricity and orbital inclination), the geosynchronous orbit can offer a much more flexible satellite than the low earth orbit, such as figure-8-like and some other complex tracks. However, ultra-high orbit makes many classical approximations unsuitable and means more complex challenges in SAR imaging processing.

Due to the asymmetric satellite flight trajectory within the synthetic aperture time in the GEO SAR system, when the point target elevation is non-zero within the irradiation 
scope, there are no two points with the same slant range history [4]. At the same time, the azimuth Doppler frequency modulation (FM) rate depends on the second derivative of the slant range, so it is also related to the target elevation. Therefore, in the process of GEO SAR imaging, except for the reference point, other points will be mismatched in the azimuth compression process $[5,6]$.

At present, most of the research on spatial variant compensation in GEO SAR is focused on the two-dimensional (2D) spatial variant error, and there are few studies on elevation spatial variation. To compensate for spatial variances in both range and azimuth directions, $\mathrm{Li}$, D. et al. proposed the improved omega $(\omega \mathrm{K})$ algorithm and three-time azimuth chirp scaling (3ACS) algorithm [1]. Both of these algorithms ignore the space variation of elevation direction. In a few papers, the sub-aperture method is proposed to compensate for the elevation spatial variant error in airborne SAR. The sub-aperture topography and aperture dependent (SATA) algorithm is first presented in [7] by Perna. By dividing the data into azimuth sub-apertures [8], the satellite flight trajectory can be considered to be symmetric about the zero Doppler center during the synthetic aperture time of each sub-aperture, then the imaging results within each sub-aperture are stitched together $[9,10]$. However, due to the short synthetic aperture time of each sub-aperture, the final azimuth resolution will be very poor, resulting in an increase in phase error within each sub-aperture. There is also great difficulty in stitching; that is, a small splicing error will lead to a large phase error. In order to compensate for the elevation spatial variant error without reducing the resolution of the imaging results, an improved range doppler azimuth chirp scaling (RD-ACS) algorithm is proposed to compensate for the elevation spatial variant error for the slow variant slope. However, the algorithm has some limitations; the focusing effect is still poor for complex scenes with a fast variant part [11]. On the other hand, current elevation inversion is a mostly repeat-pass interference system, but the process requires two coherent imaging results, and to obtain the ideal interference results is a complicated process [12]. This is difficult to realize in the design of the GEO SAR system. Therefore, it is necessary to propose a feasible elevation inversion method based on GEO SAR.

To address these deficiencies, elevation spatial variant error compensation and elevation inversion of the fast variable part are considered based on the previous research results in this paper. A complex scene can be divided into a slowly variant slope and the remaining fast variant part. For the slope, the improved RD-ACS algorithm can be used for the first step of compensation, and the coarse-focused result can be obtained. For the remaining part, since the elevation spatial variant error phase can be fitted by a polynomial, and the second-order polynomial fitting is accurate enough in general. Therefore, the map-drift (MD) algorithm can be used for compensation. The MD algorithm estimates quadratic phase error (QPE) of raw data according to the displacement between different sub-aperture images. If the synthetic aperture time is too long or the target is high, there may be a high-order phase error remnant, so the phase gradient autofocus (PGA) algorithm can be used for self-focusing compensation again. The PGA algorithm retrieves high-order terms of phase error by exploiting the phase gradient of prominent scatters, so its performance is dependent on the number of prominent scatters and is generally affected by noise and clutter. Hence the MD algorithm is usually more robust than the PGA algorithm when there is a lack of prominent targets in the scene. Therefore, combining the advantages of the two algorithms, we propose a block-MD-PGA algorithm for fine focusing. The imaging region was divided into several sub-blocks along with the range and azimuth direction. In each of these sub-blocks, the second-order phase error is compensated, and the error coefficient is obtained by using the MD algorithm $[13,14]$. Then, the residual high-order error was compensated by the PGA algorithm [15-18]. In each sub-block, the target with the highest scattering intensity in each range cell is selected as a reference. On the basis of MD algorithm compensation, the high precision compensation effect can be realized by several iterations of the PGA algorithm. By using the obtained error coefficient, the target elevation can be obtained according to the relation between the elevation and the coeffi- 
cient. This method can be considered an alternative to other methods intended to obtain an absolute three-dimensional (3D) position from SAR acquisitions. The algorithm can be applied to dot-matrix targets well. The algorithm also has a good compensation effect when the targets elevations in each sub-block are almost the same.

This paper is organized as follows. Section 2 establishes the 3D scene image geometric model and introduces the source of the elevation spatial variant error. Section 3 analyzes the spatial variant phase error. The phase error caused by the elevation spatial variation can be accurately fitted by a second-order polynomial. In Section 4, on the basis of the coarse-focused results obtained by the improved RD-ACS algorithm, the compensation of the residual fast variant error is mainly analyzed, and a block-MD-PGA algorithm is conducted to achieve fine focusing. By utilizing the relationship between the elevation and the spatial variant error coefficient, the target elevation information can be inverted. Then, the accuracy of elevation inversion is analyzed. Section 5 performs the simulation experiments of dot-matrix targets and targets based on the high-resolution TerraSAR-X image to validate the proposed method. Sections 6 and 7 conclude the whole work, including a discussion on future research.

\section{Geometric Model}

We firstly established a classical SAR imaging geometric model, as shown in Figure 1 below. The inclination of the slope is $\alpha$, point $P_{t}$ is located on the slope and the height of the target $P_{t, h}$ relative to $P_{t}$ is $h, P_{t, c}$ represents the corresponding point of $P_{t, h}$, that is, at the zero Doppler center moment, the shortest slant range between the two points is the same, and the solution of the corresponding point can be referred to in [11]. When the improved RD-ACS algorithm is used for imaging, the slant range history of $P_{t, h}$ is replaced by the slant range history of $P_{t, c}$. The trajectory of the satellite is asymmetric about the zero Doppler plane, so there are differences in the slant range history between the two points. The slant range history plays a significant role in the imaging process, so the differences will make the focusing performance of the imaging results worse. In particular, the Doppler frequency modulation (FM) is related to the second partial derivative of the slant range, which results in the mismatching of the azimuth chirp rate, resulting in serious azimuth defocus. The following formula is derived for the slant range history error.

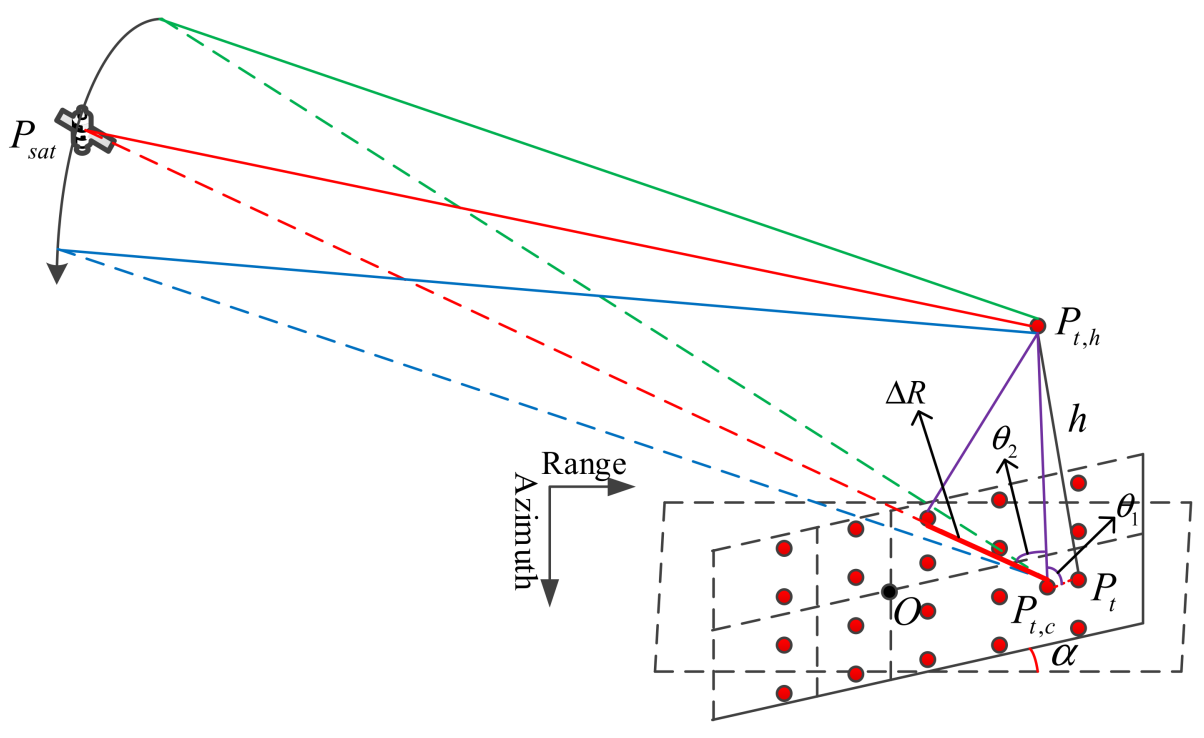

Figure 1. SAR geometry model.

The coordinate vector of the satellite position is expressed as $\vec{P}_{\text {sat }}\left(a, i, e, \Omega, W, f\left(t_{a}\right)\right)$, which can be solved by six orbital elements (semi-major axis, orbital inclination, eccentricity, 
right ascension of ascending node, argument of perigee and true anomaly). Then the slant range history error between the target and its corresponding point can be expressed as:

$$
\Delta R\left(t_{a} ; t_{0}, R_{0} ; h\right)=\left|\overrightarrow{P_{s a t} P_{t, h}}\right|-\left|\overrightarrow{P_{s a t} P_{t, c}}\right|=R\left(t_{a} ; t_{0}, R_{0} ; h\right)-R^{\prime}\left(t_{a} ; t_{0}, R_{0} ; h\right)
$$

where $t_{a}$ is the azimuth time, $t_{0}$ represents the time for the beam center points to the target, $R_{0}$ is the slant range at the moment $t_{0} . R\left(t_{a} ; t_{0}, R_{0} ; h\right)$ and $R^{\prime}\left(t_{a} ; t_{0}, R_{0} ; h\right)$ are the slope range history of the target and the corresponding point. When $t_{a}$ is equal to zero, $\Delta R$ is also zero. The phase history error can be obtained as follows.

$$
\Delta \phi\left(t_{a} ; t_{0}, R_{0} ; h\right)=\frac{4 \pi \cdot \Delta R\left(t_{a} ; t_{0}, R_{0} ; h\right)}{\lambda}
$$

where $\lambda$ is the wavelength. It can be concluded that the trajectory of the satellite in the GEO SAR system is asymmetrical about the zero Doppler plane, which leads to the deviation of the slant range between the target at a certain height and its corresponding point, thus causing the phase error in imaging processing.

\section{Analysis of the Elevation Spatial Variant Error}

Since the orbit altitude of GEO SAR is about $36,000 \mathrm{~km}$, a more accurate slant range model is necessary, and the second-order slant range model in geosynchronous orbit is no longer applicable [19-21]. In this paper, we use the fifth-order Taylor expansion slant range model, and the accuracy is sufficient [11]. The slant range model formula is as follows.

$$
R\left(t_{a}\right)=R_{0}+k_{1} t_{a}+k_{2} t_{a}^{2}+k_{3} t_{a}^{3}+k_{4} t_{a}^{4}+k_{5} t_{a}^{5}
$$

The coefficients $k_{i}, i=1,2,3,4,5$ in the slant range model can be directly fitted. Since the slant range history is related to the target elevation, the model coefficient is also affected by the elevation. Then, we will analyze the elevation information's influence on the phase error. There are two sources of the phase error; part of the error is caused by the difference in the slant range history of the target and its corresponding point, which is the dominant factor and will be analyzed in the next chapter, the other part is caused by the difference between the slant range history of the fifth-order Taylor expansion model and the real one. It has been analyzed in [11] that the phase difference caused by the fifth-order fitting (corresponding to the difference of the slant range history) can be ignored. Therefore, this part of the error has almost no impact on the imaging quality, and will not be discussed in this paper.

Since the slant range history between the target and the corresponding point can be fitted by the fifth-order Taylor expression, the expression of $\Delta \phi\left(t_{a} ; t_{0}, R_{0} ; h\right)$ can be derived by combining Equations (2) and (3).

According to Equation (4), the phase error changes with the central moment $t_{0}$, the shortest slant range $R_{0}$ and the azimuth time $t_{a}$ under different elevation conditions can be drawn, and the results are shown in Figure 2.

$$
\Delta \phi\left(t_{a} ; t_{0}, R_{0} ; h\right)=\frac{4 \pi}{\lambda} \cdot\left(\Delta R_{0}+\sum_{i=1}^{5} \Delta k_{i} t_{a}^{i}\right)
$$

As can be seen from Figure 2, the synthetic aperture time is $435.6 \mathrm{~s}$, different central moments and different shortest slant ranges have little influence on the phase error change, but when the target elevation is increased, the error changes greatly. The target located at the scene center is analyzed below. 

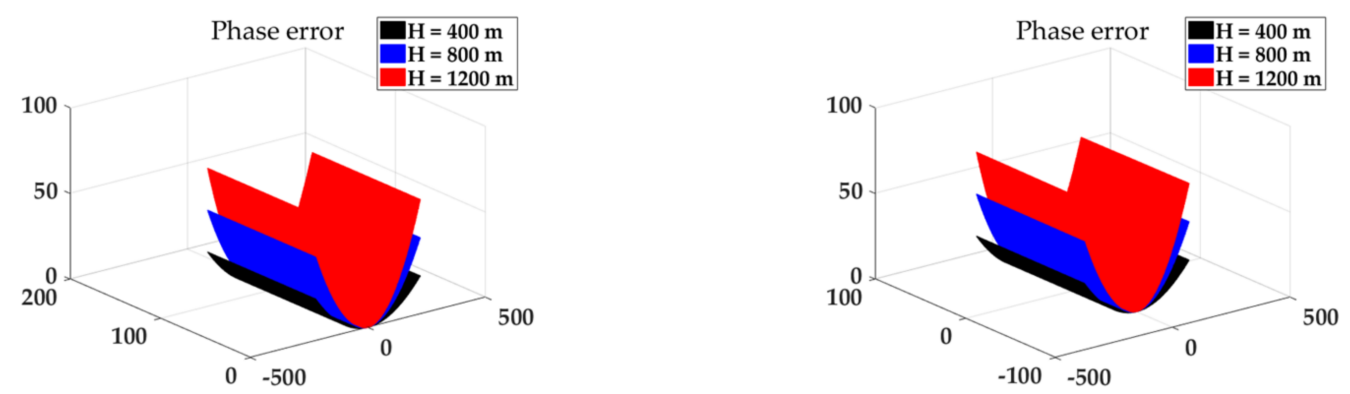

Figure 2. The variation curve of phase error with a central moment, shortest slant range and azimuth time at different elevations.

As can be seen from Figure 3, the phase error increases with the increase of the target elevation, and the second-order polynomial has a good fitting effect on the phase error represented by the fifth-order Taylor expansion slant range model. Therefore, the phase error can be rebuilt as Equation (5).

$$
\Delta \phi\left(t_{a} ; t_{0}, R_{0} ; h\right)=\frac{4 \pi}{\lambda} \cdot\left(\Delta R_{0}+\Delta k_{1} \cdot t_{a}+\Delta k_{2} t_{a}^{2}\right)
$$
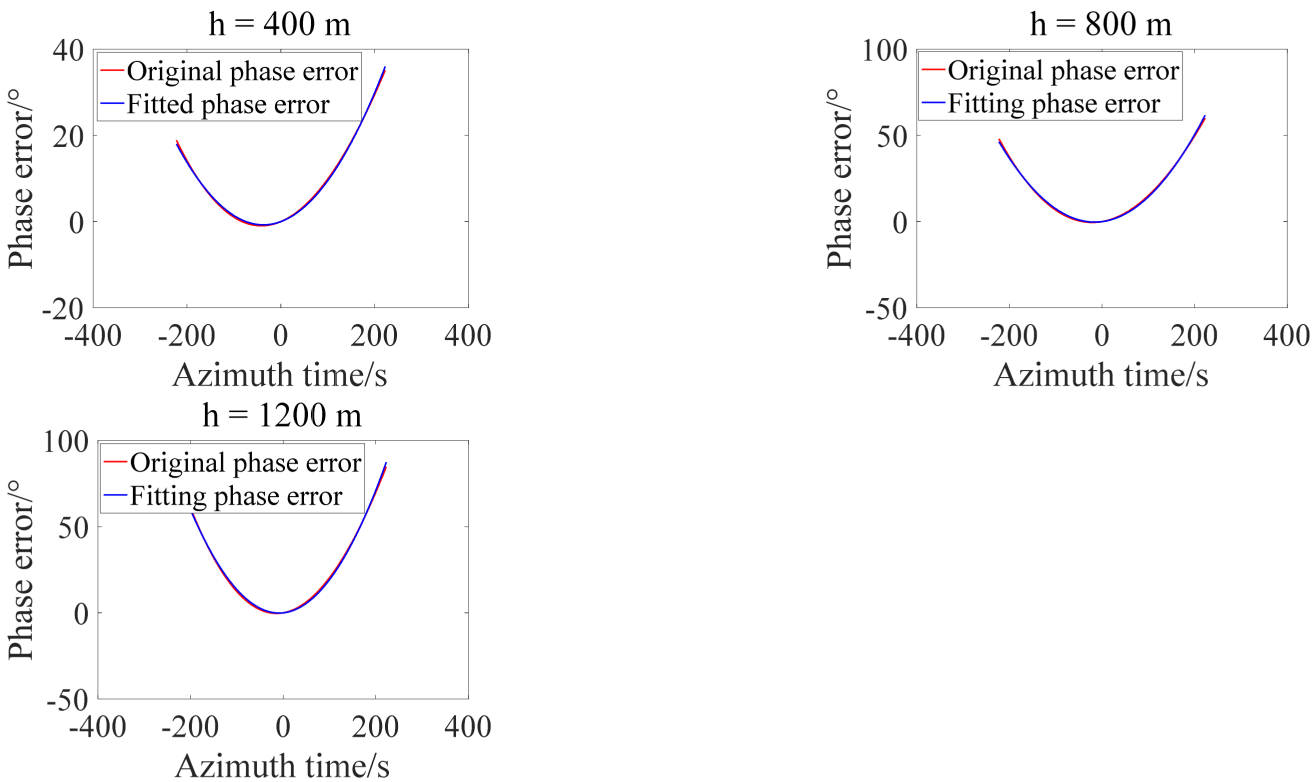

Figure 3. The original and second-order fitted phase error.

The imaging result of the point target at $h=600 \mathrm{~m}$ is given below, and the variation curve of imaging performance parameters with $h$ is drawn, as shown in Figure 4.

As can be seen from Figure 4, elevation information has a serious impact on the imaging results, and performance parameters decrease with the increase of elevation. 

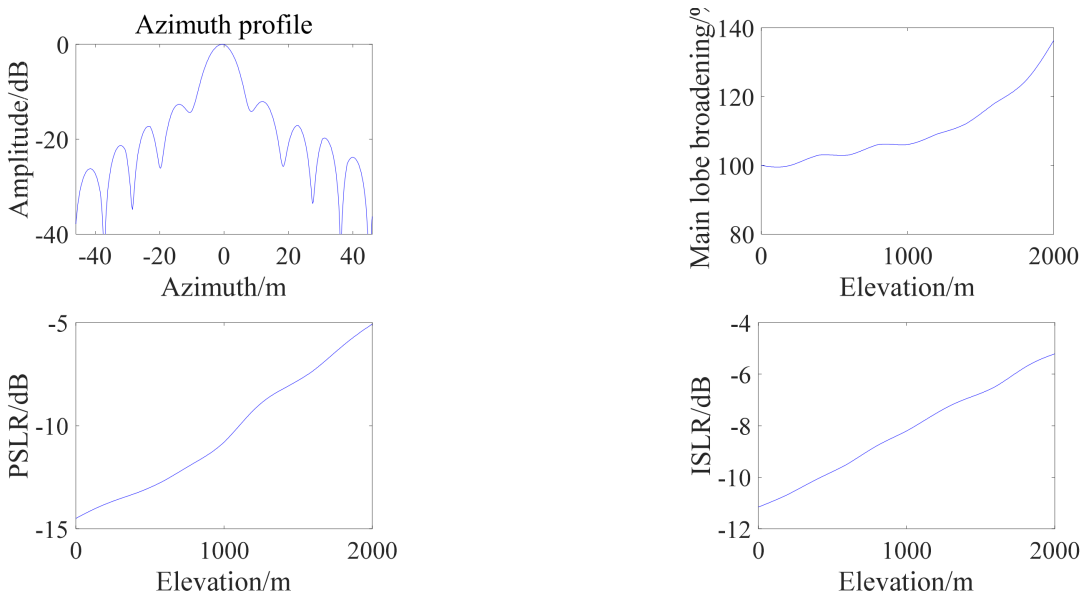

Figure 4. The imaging result with $h=600 \mathrm{~m}$ and the imaging performance curves with elevation. Note: PSLR, peak side lobe ratio; ISLR, integral side lobe ratio.

\section{Elevation Spatial Variant Error Compensation and Elevation Inversion}

In this part, we mainly compensate for the spatial variant error and inverse the elevation. For a complex scene, it can be broken down into two parts, namely, a slope and the fast variant part. In order to compensate for the decorrelation problem caused by the elevation spatial variation, a "two-step" method is proposed to achieve accurate focusing. Firstly, we use the improved RD-ACS algorithm to perform the first step of the coarse compensation for the slope. Then, the remaining part is compensated precisely by the autofocus method. Finally, according to the phase error obtained by the autofocus method, the elevation from the target to the slope is inversed, and its estimation accuracy is analyzed. The research in this part follows the steps in Figure 5. 


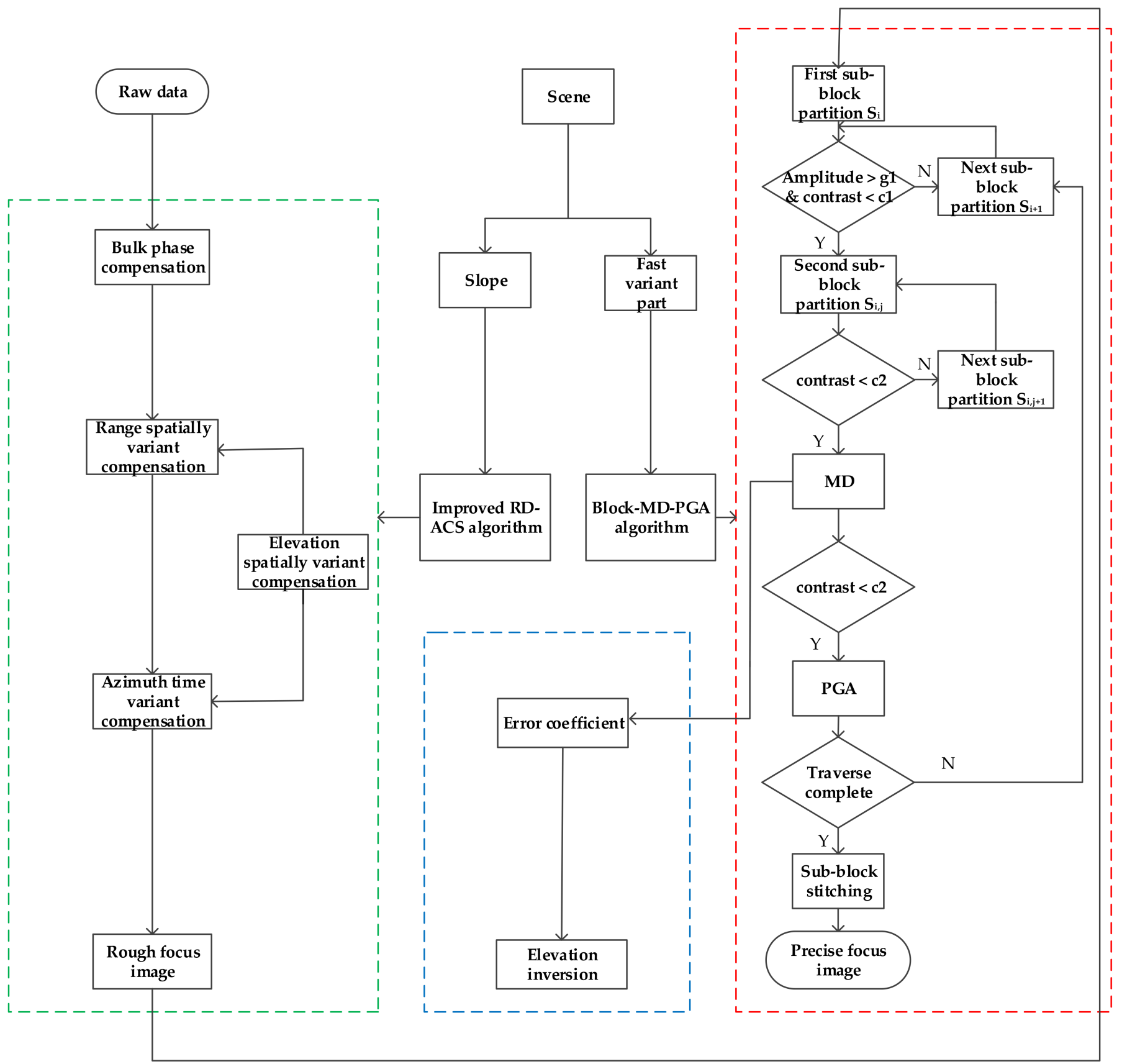

Figure 5. Algorithm flow for spatial variant error compensation and elevation inversion.

\subsection{Elevation Spatial Variant Error Compensation}

For a complex scene, it is easy to divide it into the slope and fast variant parts. The improved RD-ACS algorithm can be used for the imaging of the slope to achieve the first step of compensation. In the GEO SAR system, the rotation of the earth is taken into account, resulting in the constant change of the target coordinates over time. In the improved RDACS algorithm, the coordinates can be considered as the earth-centered fixed coordinate system, and the orbit of the satellite is elliptical in the ultra-long synthetic aperture time. The slant range history must be expressed by the fifth-order Taylor expansion. Detailed formula derivation and processing steps can be referred to in [11]. The focus of this paper is the compensation of the fast variant part.

Next, we compensate for the spatial variant error caused by the remaining fast variant part. Since the elevation variation of this part is irregular, it is impossible to break down 
the elevation spatial variation, so a block-MD-PGA algorithm is proposed to compensate. The following is a detailed description of the algorithm's processing flow.

As shown in Figure 5, the first sub-block partition is performed after coarse focus. Because there are large areas in the simulated dot-matrix targets that have no target, some areas focus well. Therefore, the purpose of the first sub-block division is to reduce the calculation amount and divide the whole image into several larger sub-blocks along the range and azimuth directions. Subsequent compensation is made only for areas with a poor focusing effect. After dividing into several large sub-blocks, the amplitude threshold $g 1$ and the contrast threshold $c 1$ are set so that the sub-blocks containing poorly focused targets can be detected. For the simulated dot-matrix targets, the amplitude threshold $g_{1}$ is defined as $60 \%$ of the maximum scattering intensity in the sub-block, and the contrast represents the imaging quality. Combining the sub-block where the point target with the well-focusing effect is located (if we can get a well focused image of this sub-block), the contrast threshold $c_{1}$ can be set as $60 \%$ of the sub-block contrast. When it is below the contrast threshold, it indicates that the sub-block has a poor focusing effect. The contrast $c_{i}$ definition after amplitude normalization in sub-block $S_{i}$ is shown in Equation (6). The number of targets in sub-block $S_{i}$ is $N_{i}$, and the pixels at each target position satisfy the conditions, as shown in Equation (7). When the conditions shown in Equation (8) are met, the sub-block $S_{i}$ contains targets and the general focusing effect of the sub-block is poor. The second sub-block is divided according to the number of targets in the sub-block $S_{i}$, Such that each sub-block contains only one target. However, for distributed targets, since there are many scattering targets in each sub-block after the first sub-block division, the second division is meaningless. We only need to ensure that the targets elevations in each sub-block are almost the same. Of course, this also has certain limitations on the application scenes. If we do not have a well-focused image of the sub-block for the dot-matrix or the distributed targets, we can also select only the sub-block of interest for subsequent compensation processing without the need for contrast threshold detection.

$$
\left\{\begin{array}{l}
J(q, k)=\frac{I(q, k)}{I_{\max }} \\
c_{i}=\frac{\sigma\left[(q, k)^{2}\right]}{E\left[J(q, k)^{2}\right]}
\end{array}\right.
$$

where $q, k$ denotes the position of the pixel in the azimuth and range directions, $I(q, k)$ represents the pixel amplitude of each point in the sub-block and $I_{\max }$ represents the maximum pixel amplitude in the sub-block. $\sigma(\cdot)$ is the standard deviation, $E(\cdot)$ is the mean.

$$
\begin{aligned}
& \{J(q, k)>g 1 \\
& \{J(q, k)>J(q, k-1) \text { and } J(q, k)>J(q, k+1) \operatorname{and} J(q, k)>J(q-1, k) \text { and } J(q, k)>J(q+1, k) \\
& \left\{\begin{array}{l}
N_{i}>0 \\
c_{i}<c 1
\end{array}\right.
\end{aligned}
$$

The length of sub-aperture of the sub-block is decided by the accuracy and efficiency of the estimated phase. The efficiency of the estimation would be improved with a large sub-aperture, whereas the resolution of the estimated phase in the azimuth is reduced. On the contrary, a short sub-aperture means that the result is sensitive to the influence of noise and other factors. In general, to reach a high accuracy, we can set an overlap area between the neighboring sub-aperture, and each sub-block can also be zeroed along the azimuth, so there is no strict constraint on the length of sub-aperture.

When the conditions shown in Equations (7) and (8) are met, it indicates that there are targets with poor focusing effects in the sub-block $S_{i}$. According to the number $N_{i}$ of targets in the $S_{i}$, a small area around each target is selected for the second sub-block division. The sub-block partition schematic is shown in Figure 6. 


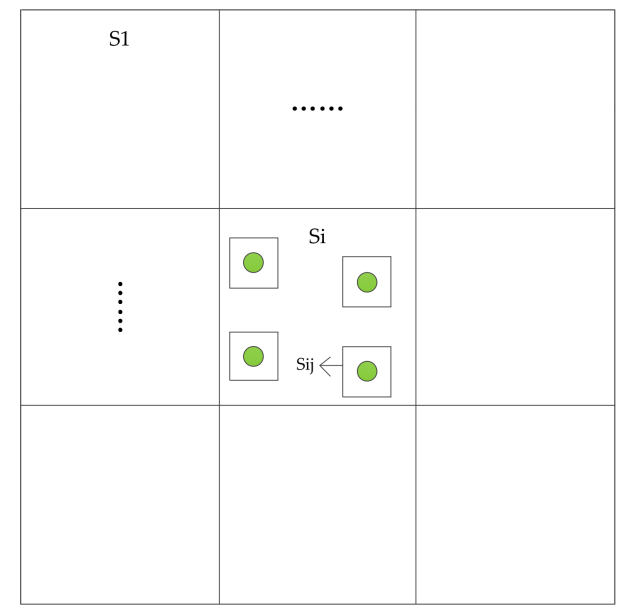

Figure 6. The sub-block partition schematic.

Then, the contrast $c_{i, j}$ of each small sub-block $S_{i, j}$ is compared. When the contrast $c_{i, j}$ is less than the contrast threshold $c_{2}$, where $c_{2}$ is defined similar to $c_{1}$, except within the subblock defined after the second partition, the autofocus method is used to compensate for the small sub-block $S_{i, j}$. According to Figure 2 in Section 3, the elevation spatial variant error can be fitted with a second-order polynomial with high accuracy. Therefore, the MD algorithm for compensating polynomial errors can be used for processing. After MD algorithm compensation, the Doppler FM error rate can be obtained, which is ready for the following elevation inversion. Then the sub-block $S_{i, j}$ was tested again for contrast. When the target height is large, the remaining high order errors in the second-order fitting may lead to an obviously asymmetric side lobe, and the contrast is still less $c_{2}$, which needs to be compensated by a PGA algorithm. For a better compensation effect, the PGA algorithm can carry out multiple loops. Then all the sub-blocks are traversed in turn, and finally, all the sub-blocks are stitched according to the initial position.

\subsection{Elevation Inversion and Estimation Accuracy Analysis}

As can be seen from Figure 1, the slant range error $\Delta R$ within the synthetic aperture time has the following geometric relationship with the target elevation.

$$
\frac{\Delta R\left(t_{a} ; t_{0}, R_{0} ; h\right)}{\cos \theta_{2}}=\frac{h}{\sin \theta_{1}}
$$

where the two angles $\theta_{1}$ and $\theta_{2}$ have been marked in Figure 1. According to Equation (2), the phase error is directly related to the target elevation. The phase error is also related to the position of the satellite on orbit, the antenna azimuth size and the incident angle, so the relationship between the phase error and the target elevation is as follows.

$$
\Delta \phi\left(t_{a} ; t_{0}, R_{0} ; h\right)=\frac{4 \pi \cos \theta_{2}}{\lambda \sin \theta_{1}} \cdot h
$$

The variation of the maximum phase error with the target elevation in the synthetic aperture time is shown in Figure 7. The parameters are shown in Table 1. 


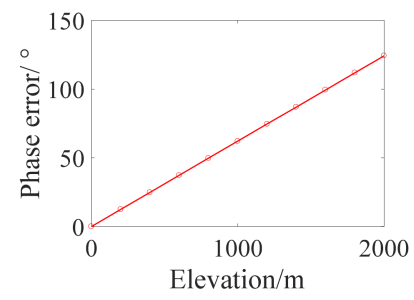

Figure 7. Relationship between phase error and elevation.

Table 1. System parameter.

\begin{tabular}{cccc}
\hline Parameter & Value & Parameter & Value \\
\hline Semi-major axis & $42,164.17 \mathrm{~km}$ & Right ascension of ascending & $115^{\circ}$ \\
Eccentricity & $1 \times 10^{-8}$ & Perigee & $270^{\circ}$ \\
Orbital inclination & $60^{\circ}$ & True anomaly & $90^{\circ}$ \\
Carrier frequency & $1.25 \mathrm{GHz}$ & Antenna size & $45 \mathrm{~m} \times 45 \mathrm{~m}$ \\
Squint angle & $0^{\circ}$ & Incident angle & $35.2^{\circ}$ \\
Pulse duration & $2.5 \mu \mathrm{s}$ & Chirp bandwidth & $30 \mathrm{MHz}$ \\
\hline
\end{tabular}

It can be seen that when the parameters are fixed, the phase error is linear with the elevation, that is, $\cos \theta_{2} / \sin \theta_{1}$ is a constant. It can be seen from Equation (5) that the phase error (slant range error) can be fitted with a second-order polynomial, so there is a linear relationship between azimuth FM rate $K_{a}$ and elevation. For the second-order expansion of the slant range model, the azimuth FM rate is typically described as:

$$
K_{a}=\frac{2 B}{\lambda R_{0}}=\frac{2\left(\dot{\vec{R}} \cdot \dot{\vec{R}}+\vec{R} \cdot R^{T}\right)}{\lambda R_{0}}
$$

where $\vec{R}, \vec{R}$ and $\vec{R}$ are the range, velocity and acceleration vectors, respectively, and $\left({ }^{T}\right)$ is the transpose [22]. Suppose a locally flat orbit has a satellite velocity $V_{\text {sat }}$ and acceleration related to the gravity at the satellite's height $g_{H s}$. The velocity parameter can be approximated as:

$$
B \approx V_{s a t}^{2}+R_{0} \cdot g_{H s} \cdot \cos \theta_{l k}
$$

where $\theta_{l k}$ is the look-down angle. By referring to Figure 8 , the geometric model of elevation $h$ can be established.

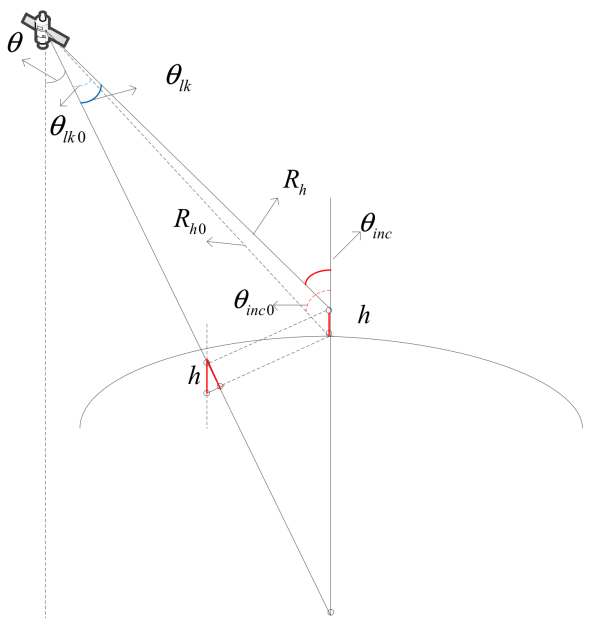

Figure 8. The geometric model of elevation $h$. 
where $\theta_{l k 0}$ and $\theta_{i n c 0}$ represent the look-down angle and incidence angle at zero elevation, respectively, while $\theta_{l k}$ and $\theta_{i n c}$ represent the look-down angle and incidence angle when elevation is $h$, respectively, $R_{h 0}$ and $R_{h}$ indicate the shortest slant range if the target elevation is zero, respectively, $\theta$ is the angle between the range vector from the satellite to the earth center and the range vector from the earth center to the target. Therefore, the FM rate error can be approximated as:

$$
\begin{aligned}
\Delta K_{a} & \approx \frac{2 g_{H s}}{\lambda R_{0}} \cdot\left(R_{h 0} \cdot \cos \theta_{l k 0}-R_{h} \cdot \cos \theta_{l k}\right)=\frac{2 g_{H s}}{\lambda R_{0}} \cdot\left(R_{h 0} \cdot \cos \theta_{i n c 0}-R_{h} \cdot \cos \theta_{i n c}\right) \\
& =\frac{2 g_{H s}}{\lambda R_{0}} \cdot h \cdot \cos \theta
\end{aligned}
$$

where $g_{H s}$ can be solved by the law of universal gravitation. Then the target elevation can be retrieved by the following formula.

$$
h \approx \frac{\lambda R_{0}}{2 g_{H s} \cdot \cos \theta} \cdot \Delta K_{a}
$$

where $\Delta K_{a}$ can be obtained by the MD algorithm mentioned above. The accuracy of elevation is analyzed below. The azimuth sub-band time shift can be expressed by:

$$
\Delta t_{a z}[n]=\beta \cdot f_{a z}[n], n=0,1
$$

where $f_{a z}[n]$ is the azimuth center frequency of the each of these sub-bands and $\beta$ is the constant defined as $\beta=\Delta K_{a} / K_{a}^{2}$. The azimuth frequency is given by:

$$
f_{a z}[n]=-\frac{B_{a}}{2}+\frac{B_{a}}{4}+\frac{B_{a}}{2} \cdot n, n=0,1
$$

where $B_{a}$ is Doppler bandwidth. According to [23], $\beta$ can be estimated with the following accuracy:

$$
\sigma_{\beta}=\frac{\sigma_{\Delta t_{a z}}}{\sqrt{\left(f_{a z}[0]\right)^{2}+\left(f_{a z}[1]\right)^{2}}}
$$

The azimuth time positioning accuracy for each sub-band is given by [24]:

$$
\sigma_{\Delta t_{a z}}=\sqrt{\frac{3}{2}} \cdot \frac{2 \sqrt{2}}{\pi B_{a} \sqrt{S C R}}=\frac{2 \sqrt{3}}{\pi B_{a} \sqrt{S C R}}
$$

where SCR is signal-to-clutter ratio. $R_{0}$ can be substituted by $H_{\text {orbit }} / \cos \theta_{\text {inc }}, H_{\text {orbit }}$ is the orbital altitude of the satellite. Thus, taking into account the gravity at the satellite's height and putting all the equations together, the height accuracy is given by:

$$
\sigma_{h}=\frac{2 \cdot V_{\text {sat }}^{4} \cdot \cos \theta_{\text {inc }} \cdot\left(R_{\text {earth }}+H_{\text {orbit }}\right)^{2}}{M \cdot G \cdot \lambda \cdot H_{\text {orbit }} \cdot \pi \cdot B_{a}^{2} \cdot \cos \theta} \cdot \sqrt{\frac{288}{3 \cdot S C R}}
$$

where $R_{\text {earth }}$ is the Earth radius, $M$ is the total Earth mass and $G$ is the gravitational constant. According to Equation (19), SCR and incident angle can be increased to improve the estimation accuracy. Doppler bandwidth and antenna size is also related. Next, we draw the curve of the theoretical elevation estimation accuracy with the SCR for different incidence angles $\theta_{i n c}$ and different antenna azimuth sizes $L_{a}$, as shown in Figure 9. 


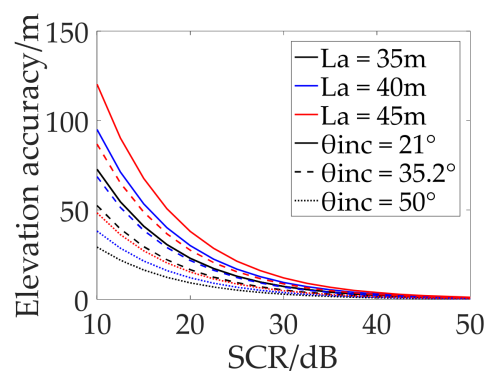

Figure 9. Theoretical elevation accuracy depending on SCR for different incidence angles and different antenna sizes.

It can be seen that reducing the antenna azimuth size can also improve the accuracy of the elevation accuracy.

\section{Simulations}

We arrange a $5 \times 5$ dot-matrix target on a slope with an angle $\alpha=36.7^{\circ}$, and the intervals between adjacent points along the range and azimuth direction are 287 and $104 \mathrm{~m}$, respectively. Among them, two points $P_{1}$ and $P_{2}$ in the lattice have a distance of 660 and $1150 \mathrm{~m}$ from the slope, respectively, as shown in Figure 10.

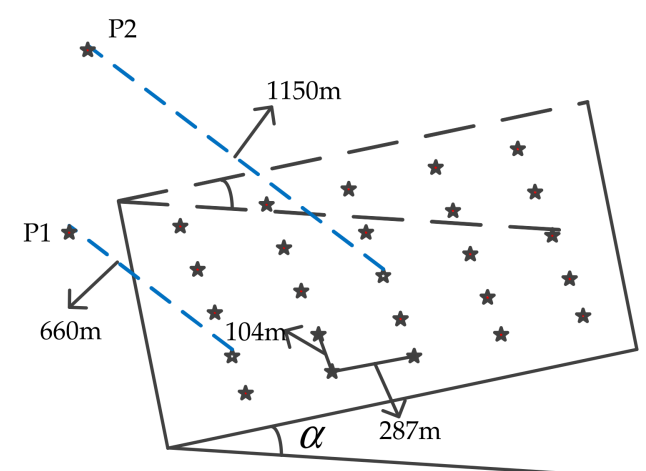

Figure 10. Scene geometry model.

The improved RD-ACS algorithm is used for the first step of imaging, and the imaging results are shown in Figure 11 below.

It can be seen that the two points $\mathrm{P} 1$ and $\mathrm{P} 2$, are separated from the slope, resulting in a poor focusing performance. Moreover, the height of P2 is larger, and the defocus is more serious. Next, we process the rough focusing image by block-MD-PGA algorithm in the second step. After sub-block partitioning and contrast comparison, it is found that the region near the points $\mathrm{P} 1$ and P2 needs autofocus compensation. The compensation results of the first MD algorithm are shown in Figure 12, and the azimuth FM rate error $\Delta K_{a}$ is obtained, as shown in Table 2.

Table 2. Statistical analysis for the known height.

\begin{tabular}{ccc}
\hline P1 & P1 & P2 \\
\hline FM error $\left(1 \times 10^{-6}\right)$ & 29.01 & 53.65 \\
True height $(\mathrm{m})$ & 660 & 1150 \\
Inversion height $(\mathrm{m})$ & 646.45 & 1139.95 \\
Estimation error $(\mathrm{m})$ & 13.55 & 10.05 \\
Theoretical accuracy $(\mathrm{m})$ & 27.48 & 27.48 \\
\hline
\end{tabular}




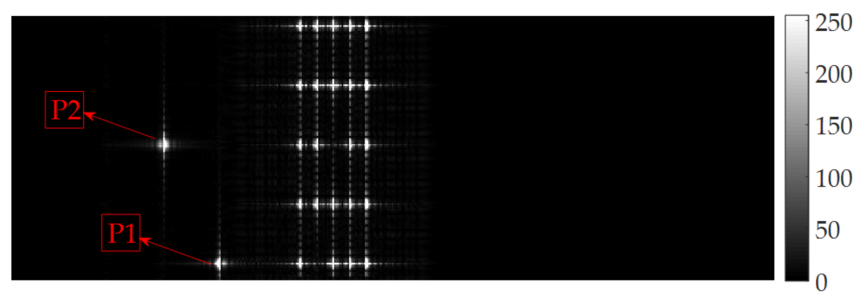

(a)

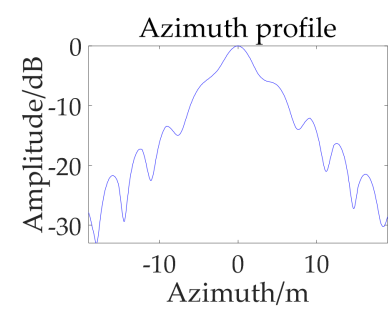

(b)

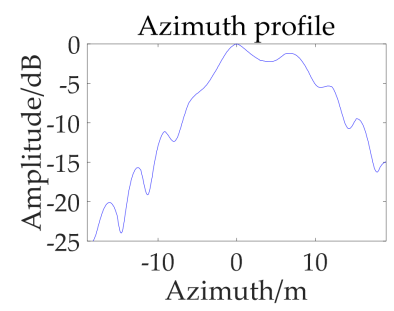

(c)

Figure 11. (a) Imaging result after the first step; (b) Azimuth profile of point P1; (c) Azimuth profile of point P2.

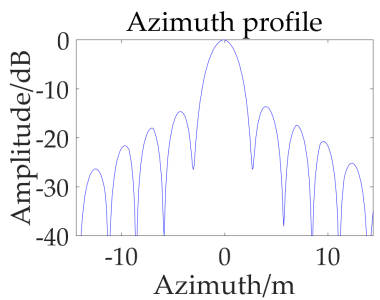

(a)

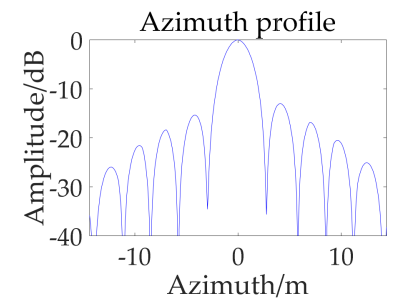

(b)

Figure 12. Imaging results after MD algorithm compensation. (a) Azimuth profile of point P1; (b) Azimuth profile of point P2.

It can be seen that after the MD algorithm compensation, the focusing performance is significantly improved, but an obvious asymmetric side lobe phenomenon will appear, and the larger the target height is, the more obvious the phenomenon will be, which means the peak side-lobe may be greater than $-13 \mathrm{~dB}$, so that the weak target is covered by the nearby strong target. Next, the PGA algorithm is used to compensate for the area near the two points; this was repeated 10 times. The results after compensation and performance analysis of each step are shown in Figure 13 and Table 3.

Table 3. Performance parameters.

\begin{tabular}{ccccc}
\hline & Point & IRW & PSLR (dB) & ISLR (dB) \\
\hline \multirow{2}{*}{ After first step } & P1 & 1.2188 & -11.6457 & -8.4702 \\
& P2 & 1.2417 & -9.9542 & -6.8024 \\
After MD algorithm & P1 & 1.0313 & -13.1346 & -10.3346 \\
\multirow{2}{*}{ PGA iteration 3 times } & P2 & 1.0313 & -12.7472 & 10.3378 \\
\multirow{2}{*}{ PGA iteration 5 times } & P1 & 1.0313 & -13.3301 & -10.3011 \\
\multirow{2}{*}{ PGA iteration 10 times } & P2 & 1.0313 & -12.7632 & -10.3724 \\
& P1 & 1.0313 & -13.4006 & -10.2912 \\
& P2 & 1.0313 & -13.2143 & -10.3781 \\
& P1 & 1.0313 & -13.4381 & -10.4419 \\
& P2 & 1.0313 & -13.7262 & -10.5883 \\
\hline
\end{tabular}




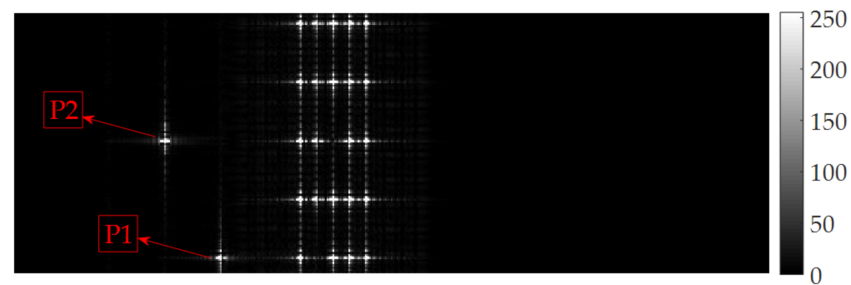

(a)

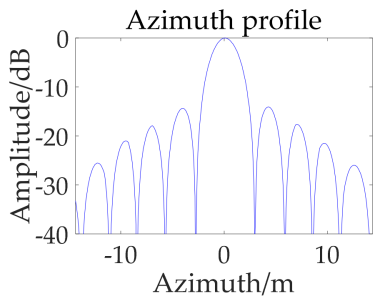

(b)

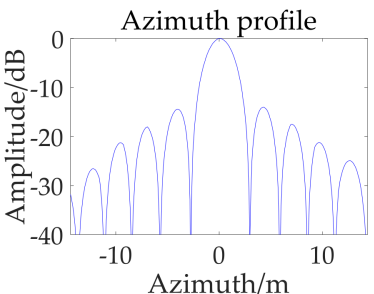

(d)

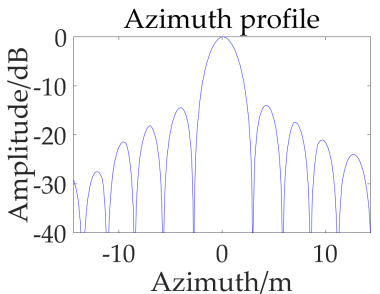

(f)

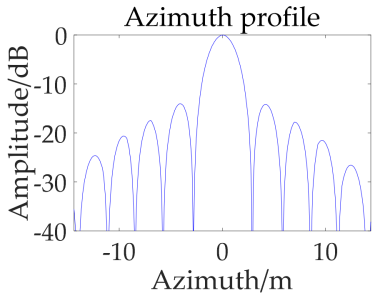

(c)

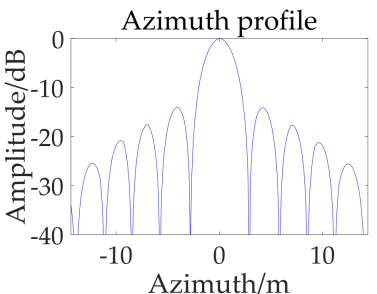

(e)

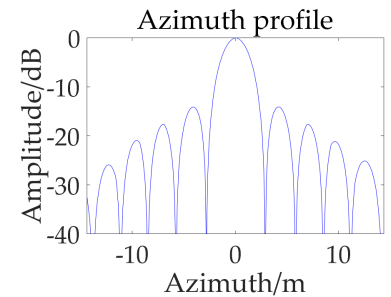

(g)

Figure 13. (a) Imaging result after 10 iterations; (b,d,f) are the azimuth profiles of 3,5 and 10 iterations of PGA at point P1; (c,e,g) are the corresponding azimuth profiles at point P2.

It can be seen that after the compensation of the second step, the focusing effect has been very effective, and the focusing effect is better as the iterations increase. Then, according to the $\Delta K_{a}$ obtained by the MD algorithm in the second step of processing, the elevation inversion is carried out. We set SCR as $20 \mathrm{~dB}$ and draw the actual curve of $\Delta K_{a}$ with the elevation according to the FM rate error by the MD algorithm. Then we plot a fitted straight curve that varies with elevation according to Equation (13) in Figure 14.

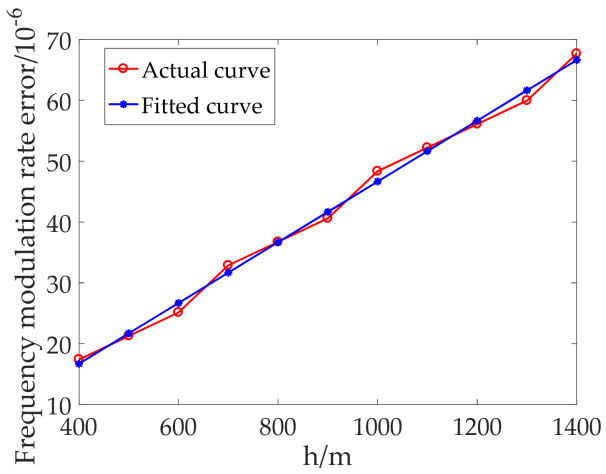

Figure 14. FM rate error varies with the elevation. 
It can be seen that the measured FM rate error is almost consistent with the theoretical analysis. Next, the two points with an elevation of 660 and $1150 \mathrm{~m}$ are used to inverse the target elevation according to the fitted linear. Elevation inversion results are shown in Table 2.

As can be seen in Figure 9, when the parameters shown in Table 1 are used, and the SCR is set to $20 \mathrm{~dB}$, the theoretical elevation inversion accuracy is $27.48 \mathrm{~m}$. It can be concluded that the spatial variant error introduced by the elevation leads to the mismatching of the azimuth FM rate, and the mismatching error is proportional to the elevation, which can be used to accurately inverse the target elevation. Then, further experiments are carried out based on the high-resolution TerraSAR-X image acquired near the Baoan, Shenzhen, China. We selected a stadium from the image and selected 13 strong scattering points on it. In order to better display the defocus phenomenon, we set the elevation of these points as $550 \mathrm{~m}$ to carry out echo simulation, an image with the improved RD-ACS algorithm, and finally, use the block-MD-PGA algorithm proposed in this paper to compensate. Figure 15 shows the SAR image of the stadium, the final compensation result of the scattering points and the performance analysis of a randomly selected point P3 before and after compensation.

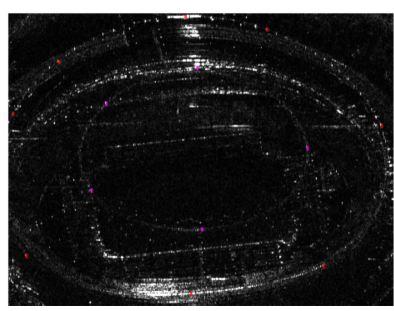

(a)

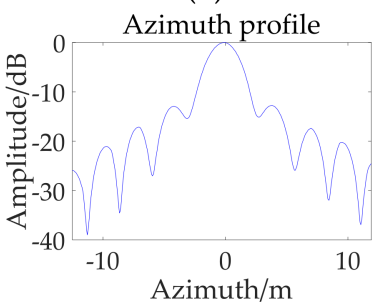

(c)

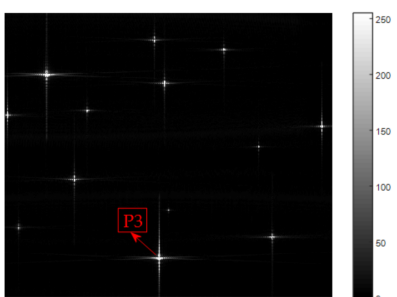

(b)

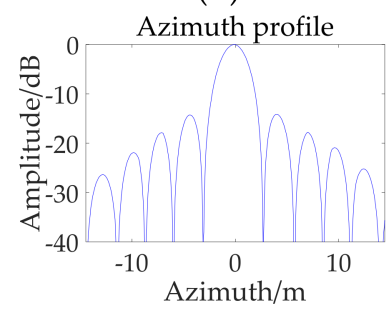

(d)

Figure 15. (a) Stadium with scattering points; (b) Final compensation result of the scattering points; (c) Azimuth profile of P3 before compensation; (d) Azimuth profile of P3 after compensation.

The parameters of IRW, PSLR and ISLR before compensation are 1.0625, -11.9588 and $-8.8544 \mathrm{~dB}$, respectively, and the results after compensation are $1.0313,-13.7064$ and $-10.3445 \mathrm{~dB}$, respectively. The elevation obtained by phase error inversion is $545.32 \mathrm{~m}$. Finally, in order to obtain the applicable conditions of the autofocus algorithm, we draw the curve of residual phase error with the variation of signal-to-noise ratio (SNR). In the process of autofocus compensation, the MD algorithm is necessary, and elevation inversion is also carried out according to the FM rate obtained by the MD algorithm. Therefore, only residual phase analysis obtained by the MD algorithm is enough. We selected the target elevation of the points at 400 and $800 \mathrm{~m}$ for analysis, and the results are shown in Figure 16.

It can be seen that for the point target, even if the SNR deteriorates to $-5 \mathrm{~dB}$, the residual phase error is still controlled under $45^{\circ}$, and the error almost does not vary with the SNR. The results would be easily accepted with a further explanation. Assuming that the map drift obtained with the autofocus method can be expressed as

$$
\Delta K^{\prime}=\Delta K+\Delta K_{n}
$$

where $\Delta K^{\prime}$ is the FM rate error of the image with the influence of different SNRs, $\Delta K$ is the FM rate error to be solved and $\Delta K_{n}$ is the FM rate error of the noise. Considering 
the noncorrelation of the noise, $\Delta K_{n}$ obtained by MDA can be approximated to zero. It can also be seen that the residual phase error increases with the target elevation. This is because the MD algorithm cannot compensate for the high-order polynomial error, and the residual high-order polynomial error increases as the target elevation increases. When the error reaches a certain degree, the PGA algorithm is needed to compensate. Even if the target is very high, the error is within the range that can be compensated by the PGA algorithm. This is consistent with the previous analysis and simulation results. Therefore, we can conclude that the proposed algorithm can work well in the low SNR cases and is almost independent of SNR.

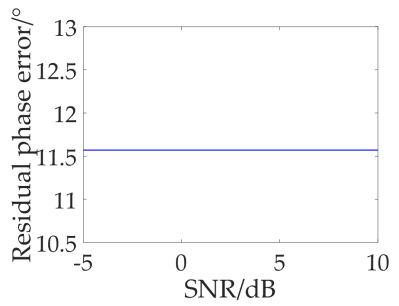

(a)

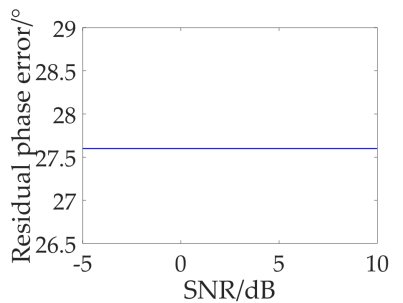

(b)

Figure 16. Residual phase error varies with SNR; (a) Target elevation is $400 \mathrm{~m}$; (b) Target elevation is $800 \mathrm{~m}$.

\section{Discussion}

In this paper, we mainly study the elevation spatial variant error compensation and elevation inversion of the fast variant part. Firstly, the source of the error is explained intuitively through the established geometric model. Then we analyze the error sufficiently and draw the conclusion that the error can be accurately fitted by a polynomial. Based on this conclusion, an autofocus compensation algorithm is proposed, and the simulations with dot-matrix targets and targets based on the TerraSAR-X image are carried out. The correctness of phase error compensation and elevation inversion is verified by experiments. Although there is no available measured data at present, the distributed targets, highly-directive targets and isotropic-like targets are also discussed. In fact, the algorithm can be used in many scenes except for the layover of the scene, where it cannot inverse the target elevation well. The point target in each sub-block can be compensated by the block autofocus method. However, for distributed targets, there are many scattering points in each sub-block; we only need one sub-block division. In future work, we can segment the coarse-focused image along the range and azimuth directions (the block size depends on the need for precision and efficiency). Then each sub-block is processed by the autofocus method, and the resulting quadratic phase error coefficient is taken as the FM error of the sub-block intermediate position. The FM error at each pixel position is obtained by interpolating along the azimuth and range directions, respectively. Then the error phase is obtained by quadratic integration. Finally, the error phase is compensated. In this way, the elevation spatial variation error compensation and the elevation inversion can be carried out well. In order to verify the feasibility of the algorithm, we will study the distributed targets echo simulation method for complex scenes in the future and utilize interpolation to improve the processing efficiency of large amounts of data in GEO SAR.

\section{Conclusions}

In this paper, we propose a complete process to solve the elevation spatial variant problem in complex scenes and utilize the elevation spatial variant error to inverse the target elevation. We divide a complex scene into two parts, one part is a slope, and the rest is a fast variant scene. For the slope, the improved RD-ACS algorithm can achieve an excellent focusing effect. For the fast variant part, the proposed block-MD-PGA algorithm is used to compensate. According to the theoretical analysis, the elevation spatial variation 
will lead to the azimuth FM rate mismatch, and the FM rate error is proportional to the elevation. Therefore, the error obtained in the process of autofocus compensation can be used to inverse the target elevation, and the elevation inversion accuracy is analyzed. Finally, the correctness of the proposed algorithm is verified based on the dot-matrix targets and targets based on the TerraSAR-X image. The simulation results show that the proposed algorithm can compensate for the elevation variation error caused by the fast variant part well and can also inverse the target elevation well. Then we also conclude that the algorithm can work well under low SNR conditions. The analysis of this paper will be of great significance to the system design and many application fields, such as GEO SAR wide swath terrain observation.

Author Contributions: All authors have made substantial contributions to this work. F.C. and D.L. formulated the theoretical framework. F.C. designed the simulations. F.C. carried out the simulation experiments. F.C., D.L. and Z.D. analyzed the simulated data. F.C. wrote the manuscript. D.L., Y.H., Z.H. and Z.D. reviewed and edited the manuscript. Z.D. gave insightful and enlightening suggestions for this manuscript. All authors have read and agreed to the published version of the manuscript.

Funding: This research received no external funding.

Institutional Review Board Statement: Not applicable.

Informed Consent Statement: Not applicable.

Data Availability Statement: The data presented in this study are available on request from the corresponding author.

Acknowledgments: The authors would like to thank all those who gave valuable help and suggestions to this manuscript, which were essential to the outcome of this paper.

Conflicts of Interest: The authors declare no conflict of interest.

\section{References}

1. Li, D.; Wu, M.; Sun, Z.; He, F.; Dong, Z. Modelling and Processing of Two-Dimensional Spatial-Variant Geosynchronous SAR Data. IEEE J. Sel. Top. Appl. Earth Obs. Remote Sens. 2015, 8, 3999-4009. [CrossRef]

2. Bruno, D.; Hobbs, S.; Ottavianelli, G. Geosynchronous synthetic aperture radar. Acta Astronaut. 2006, 59, 149-156. [CrossRef]

3. Hu, C.; Long, T.; Liu, Z.; Zeng, T.; Tian, Y. An Improved Frequency Domain Focusing Method in Geosynchronous SAR. IEEE Trans. Geosci. Remote Sens. 2014, 9, 5514-5528.

4. Kempf, T.; Anglberger, H.; Suess, H. Depth-of-focus issues on spaceborne very high resolution SAR. In Proceedings of the IEEE International Geoscience and Remote Sensing Symposium, Munich, Germany, 22-27 July 2012; pp. 7448-7451.

5. Prats, P.; Scheiber, R.; Rodriguez-Cassola, M. On the Processing of Very High Resolution Spaceborne SAR Data. IEEE Trans. Geosci. Remote Sens. 2014, 52, 6003-6015. [CrossRef]

6. Prats, P.; Macedo, K.; Reigber, A.; Scheiber, R.; Mallorqui, J. Comprasion of topography and aperture dependent motion compensation algorithms for airborne SAR. IEEE Trans. Geosci. Remote Sens. Lett. 2007, 4, 349-353. [CrossRef]

7. Peras, S.; Zamparelli, V.; Pauciullo, A.; Fornaro, G. Azimuth-to-frequency mapping in airborne SAR data corrupted by uncompensated motion errors. IEEE Geosci. Remote Sens. Lett. 2013, 10, 1493-1497.

8. Pu, W.; Li, W.; Wu, J.; Huang, Y.; Yang, J.; Yang, H. An azimuth-variant autofocus scheme of bistatic forward-looking synthetic aperture radar. IEEE Geosci. Remote Sens. Lett. 2017, 14, 689-693.

9. Prats, P.; Scheiber, R.; Mittermayer, J.; Meta, A.; Moreira, A. Processing of sliding spotlight and TOPS SAR data using baseband azimuth scaling. IEEE Trans. Geosci. Remote Sens. 2010, 2, 770-780. [CrossRef]

10. Madsen, S. Motion compensation for ultra wide band SAR. In Proceedings of the 2001 IEEE International Geoscience and Remote Sensing Symposium (IGARSS), Sydney, Australia, 9-13 July 2015; pp. 1436-1438.

11. Chang, F.G.; Li, D.X. Elevation Spatial variation Analysis and Compensation in GEO SAR Imaging. Remote Sens. 2021, 13, 1888. [CrossRef]

12. Gray, A.; Farris, P. Repeat-pass interferometry with airborne synthetic aperture radar. IEEE Trans. Geosci. Remote Sens. 1993, 31, 180-191. [CrossRef]

13. Samczynski, P.; Kulpa, K. Coherent mapdrift techinique. IEEE Trans. Geosci. Remote Sens. 2010, 48, 1505-1517. [CrossRef]

14. Wang, G.; Zhang, M.; Huang, Y.; Zhang, L.; Wang, F. Robust Two-Dimensional Spatial-Variant Map-Drift Algorithm for UAV SAR Autofocusing. Remote Sens. 2019, 11, 340. [CrossRef]

15. Calloway, T.; Donohoe, G. Subaperture Autofocus for Synthetic Aperture Radar. IEEE Trans. Aerosp. Electron. Syst. 1994, 30, 617-621. [CrossRef] 
16. Wahl, D.; Eichel, P.; Ghiglia, D.; Jakowatz, C. Phase gradient autofocus a robust tool for high resolution SAR phase correction. IEEE Trans. Accept. Electron. Syst. 1994, 30, 827-835. [CrossRef]

17. Zhu, D.; Jiang, R.; Mao, X.; Zhu, Z. Multi-aubaperture PGA for SAR autofocusing. IEEE Trans. Aerosp. Electron. Syst. 2013, 49, 468-488. [CrossRef]

18. Wahl, D.; Jakowatz, C. New approach to strip-map SAR autofocus. In Proceedings of the IEEE 6th Digital Signal Processing Workshop, Yosemite National Park, CA, USA, 2-5 October 1994; pp. 53-56.

19. Hu, B.; Jiang, Y.; Zhang, Y.; Yeo, T. Accurate slant range model and focusing method in geosynchronous SAR. In Proceedings of the IEEE International Geoscience and Remote Sensing Symposium (IGARSS), Milan, Italy, 26-31 July 2015; pp. 4464-4467.

20. Zhao, B.; Qi, X.; Song, H.; Wang, R.; Mo, Y.; Zheng, S. An Accurate Range Model Based on the Fourth-Order Doppler Parameters for Geosynchronous SAR. IEEE Geosci. Remote Sens. Lett. 2014, 11, 205-209. [CrossRef]

21. Yu, Z.; Lin, P.; Xiao, P.; Kang, L.; Li, C. Correcting spatial variance of RCM for GEO SAR imaging based on time-frequency scaling. Sensors 2016, 16, 1091. [CrossRef] [PubMed]

22. Duque, S.; Breit, H.; Balss, U.; Parizzi, A. Absolute height estimation using a single TerraSAR-X staring spotlight acquisition. IEEE Geosci. Remote Sens. Lett. 2015, 12, 1735-1739. [CrossRef]

23. Kay, S. Fundamentals of Statistical Signal Processing: Estimation Theory; Prentice-Hall: Upper Saddle River, NJ, USA, 1998.

24. Bamler, R.; Eineder, M. Accuracy of differential shift estimation by correlation and split-bandwidth interferometry for wideband and delta-k SAR systems. IEEE Geosci. Remote Sens. Lett. 2005, 2, 151-155. [CrossRef] 